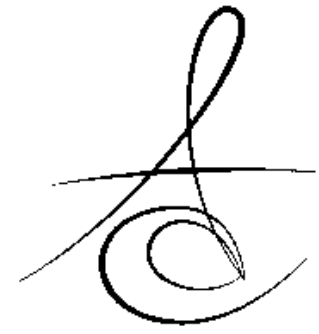

Makale Kodu/Article code: 2714

Makale Gönderilme tarihi: 24.03.2016

Kabul Tarihi; 12.04 .2016

\section{TRAVMAYA BAĞLI PERİODONTAL PROBLEMLİ VAKADA ANTERİOR ESTETİK RESTORASYON: VAKA RAPORU ${ }^{\star}$}

\section{ANTERIOR ESTHETIC RESTORATION OF THE PERIODONTALLY COMPROMISED TEETH DUE TO THE TRAUMA: CASE REPORT ${ }^{*}$}

\author{
Dt. Seda KEBAN* \\ Doç. Dr. Coşkun YILDIZ*
}

\section{öz}

Dental seramikler üstün biyolojik uyumlulukları, kimyasal stabiliteleri, renk stabilitelerini korumaları, yüzey pürüzlülüklerinin minimum olması, ağız içi sıvılardan etkilenmemeleri, çiğneme kuvvetleri ile minimum aşınmaları ve estetik özellikleri nedeniyle protetik diş hekimliğinde kullanılmaktadır. Seramiği desteklemek ve kırılmasını önlemek amacıyla başta metal alt yapı kullanılmıştır. Ancak alt yapıdan kaynaklanan metal renginin yansıması, opak görünüm, alaşımın korozyonu ve metal alerjisi nedeniyle estetik ve biyouyumluluk tam olarak sağlanamamıştır. Yapılan çalışmalar sonucunda hem seramiğin yapısı geliştirilmiş, hem de fırınlama tekniklerine bağlı olarak mekanik kuvvetlere karşı dayanıklı seramik türleri elde edilmiştir. Bu sayede tam seramik restorasyonların uygulanabilmesi mümkün kılınmıştır. Protetik diş hekimliğinin temel amacı kaybedilmiş olan fonksiyon, fonasyon ve estetiğin iade edilmesidir. Estetik problemlerin başında ise ön grup dişlere ait olan renk, şekil ve konum bozuklukları gelmektedir. Bu olgu raporunda 23 yaşında santral dişinden estetik olarak memnun olmayan bayan hastanın protetik tedavisi anlatılmaktadır. Hastanın maksiller sol santral dişinde oklüzal travmaya bağlı uzama meydana gelmiştir. Santral diş tam seramik restorasyon ile restore edilirken oklüzal travmaya bağı dişin uzamasını önlemek amacıyla sağ santral ve sol lateral dişlerin lingual bölgelerine tırnak şeklinde uzantılar hazırlanmıştır. Restorasyon lityum disilikat cam seramik ile hazırlanmış ve simantasyonu rezin siman ile yapılmıştır. Tedavi sonrasında estetik ve fonksiyon açısından başarılı sonuçlar elde edilmiş ve hasta memnuniyeti sağlanmıştır.

Anahtar Kelimeler: Oklüzal travma, İnley destekli, Tam seramik restorasyon

\section{ABSTRACT}

Due to superior biological compatibility, chemical stability, color stability, have a minimum surface roughness, not effected by oral liquids, minimum abrasion against masticatory forces and aesthetic characteristics, dental ceramics are used in prosthetic dentistry. Initially metal substructure used to support the ceramic and prevent fracture of ceramic. Aesthetic and biocompatibility are not fully ensured because of the color of the reflection from the metal substructure, opaque appearance, corrosion of alloy and metal allergy aesthetics and biocompatibility of the alloy corrosion and metal allergy fully achieved. In this way, implementation of all-ceramic restorations became possible. Both ceramic structure is developed and due to the firing technique ceramic types are obtained which is resistant to mechanical forces. The main purpose of prosthetic dentistry is restoring lost function, phonation and aesthetic. Color, shape and position disorders of anterior teeth are primary aesthetic problems. Patient is uncomfortable with discoloration of composite material and aesthetic view. In this case report, 23 years old female patient's who is not please aesthetically with central tooth, prosthetic treatment is described. Eruption of central tooth was seen due to occlusal trauma. To prevent this eruption restoration of maxillary central teeth was supported with inlay retainers of right central and left lateral teeth. Restorations were prepared with lithium disilicate glass ceramic and resin cement was used for cementation. After the treatment, successful results are obtained in terms of esthetics and function and patient satisfaction was achieved. Aesthetic needs of patient is successfully obtained by all ceramic crown restoration.

Key words: Occlusal trauma, Inlay retained, All ceramic restoration

\footnotetext{
* Marmara Üniversitesi Diş Hekimliği Fakültesi Protetik Diş Tedavisi Anabilim Dalı

₹ 5.Uluslararası Türk Prostodonti ve İmplantoloji Derneği Bilimsel Sempozyumunda poster olarak sunulmuştur(18-20 Mart 2016, Erzurum-Turkey).
} 


\section{GİRIŞ}

Aşırı oklüzal kuvvetler "dişler, destek dokular, nöromüsküler sistem, temporomandibular eklemler ve kraniofasial iskeleti içine alan çiğneme sisteminin komponentleri arasındaki fonksiyonel ilişki diye adlandırılan oklüzyonu" bozar. Bu ilişki bozulduğu zaman birçok akut ve kronik karşıt reaksiyon oluşur. Bu karşıt reaksiyonlarda doku yıkımı ortaya çıkabilir ve bu yıkıma oklüzal travma denir. ${ }^{1}$ İleri periodontal yıkımı olan hastalarda etkilenen dişlerde sıklıkla patolojik yer değiştirme meydana gelir. Periodontal desteğini kaybetmiş olan dişlerde eğilme, anterior dişlerin uzaması, dönmesi ve dişler arasında diastema meydana gelmesi gibi komplikasyonlar ile karşılaşılmaktadır. Dişlerde meydana gelen bu durumlar estetik ve fonksiyonel problemleri beraberinde getirmektedir. ${ }^{2}$

Modern diş hekimliğinde hastaların estetik beklentilerini karşılamada yüksek biyokompatibiliteye sahip tam seramik restorasyonlar gün geçtikçe önem kazanmaktadır. ${ }^{3}$ Diş hekimliğinde koruyuculuk kavramının önem kazanmasıyla minimum düzeyde diş dokusu kaldırılarak yeterli niteliklerde restorasyon hazırlanmasına yönelme olmuştur. İnley destekli restorasyonlar geleneksel köprülere göre çok daha konservatif bir tedavi yöntemidir. Ancak inley köprülerin yapımı daha fazla klinik hassasiyet gerektirmektedir. Genel olarak kabul edilmektedir ki; inley köprüler geleneksel köprülere göre diş dokusunu daha fazla korumaktadır. ${ }^{4} \mathrm{Bu}$ çalışmanın amacı santral dişinden estetik olarak memnun olmayan bayan hastanın tam seramik inley tutuculu kuron restorasyonu ile protetik olarak rehabilite edilmesidir.

\section{OLGU SUNUMU}

Marmara Üniversitesi Diş Hekimliği Fakültesi Protetik Diş Tedavisi Anabilim Dalı Kliniği'ne 23 yaşındaki bayan hasta maksiller sol santral dişinin uzamasına bağlı estetik kaybın telafisi için başvurdu. Yapılan ağız içi muayenesinde periodontal problemlere ve oklüzal travmaya bağlı olarak ilgili dişte ekstruzyon olduğu gözlendi (Resim 1). Hastanın ağız içi muayenesini takiben radyolojik değerlendirmeleri ve model analizleri yapıldı. Periodontal tedavisi tamamlandıktan sonra komşu dişlerin destek olarak kullanılmasında herhangi bir problem olmadığı görüldü. Estetik olarak durumun düzeltilebilmesi için 3 üyeli konvansiyonel köprü restorasyonu ve inley tutuculu dizayn edilen kuron restorasyon alternatifleri hastaya anlatıldı. Hasta konservatif bir yaklaşım olması sebebiyle inley tutuculu kuron restorasyon yapılmasını tercih etti. Diş preparasyonu yapılmadan önce yapılacak olan restorasyonların renk seçimi yapıldı. Destek dişlerin hazırlanması esnasında maksiller sağ santral dişin mezialinde ve maksiller sol lateral dişin mezialinde restorasyonda planlanan inley tutuculu kısımların yerleşebilmesi için $2 \mathrm{~mm}$ derinliğinde ve $3 \mathrm{~mm}$ genişliğinde kenarları yuvarlatılmış formda yuvalar hazırlandı. Ekstruze olan maksiller santral dişin preparasyonunda dişin kole bölgesinde 1-1.3 mm genişliğinde iç köşesi (aksiyal duvar ile basamak arasındaki açı) yuvarlatılmış chamfer tarzda basamak preparasyonu yapıldı. Basamak serbest dişeti kenarının $0.5 \mathrm{~mm}$ altında olacak şekilde ve dişeti konturunu takip edecek şekilde hazırlandı. Diş preparasyonu sonrası tüm keskin kenar ve köşeler yuvarlatıldı (Resim 2). İki aşamalı ölçü yöntemi ile slikon esaslı ölçü materyali (Zetaplus, Zhermack, Almanya) kullanılarak ölçü alındı ve sert alçı materyali (Elite Dental Stone, Zhermack) kullanılarak model elde edildi. Lityum disilikat cam seramik (IPS e.max Press, Ivoclar Vivadent, Schaan, Liechteinstein) materyali kullanılarak hazırlanan restorasyonların hasta ağızında provası yapıldı (Resim 3, Resim 4, Resim 5). Antagonist diş kontakları, restorasyonun rengi, protrüziv, lateral ve oklüzal ilişkiler değerlendirildi ve gerekli düzenlemeler yapıldı. Daha sonra simantasyon işlemine geçildi. Restorasyonların iç yüzeyleri 30 saniye süreyle \%5'lik hidroflorik asit (IPS Ceramic etching gel, Ivoclar Vivadent) ile asitlendi, 30 saniye süreyle hava su spreyi ile yıkandı ve hava ile kurutuldu. Daha sonra restorasyonların iç yüzeyine silan ajanı (Silane, Ivoclar Vivadent) uygulandı, 60 saniye beklendikten sonra hava ile kurutuldu. Diş yüzeyinin hazırlanmasında öncelikle prepare edilmiş diş yüzeyleri pomza ve firça yardımıyla temizlendi. Daha sonra \%37'lik fosforik asit (Total Etch, Ivoclar Vivadent) ile mine ve dentin yüzeyleri 30 saniye süreyle asitlendi ve bu süre sonunda diş yüzeyleri 30 saniye süreyle hava su spreyi ile yıkandı. Sonra sırasıyla primer (Syntac Primer, Ivoclar Vivadent), adeziv (Syntac Adhesive, Ivoclar Vivadent) ve bond (Heliobond, Ivoclar Vivadent) diş yüzeylerine uygulandı. Rezin siman (Variolink N, Ivoclar Vivadent) kullanılarak simantasyon işlemi tamamlandı. Yapıştırıcı siman artıkları dikkatlice uzaklaştırıldıktan sonra her diş için önce vestibül daha

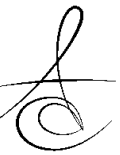


sonra da palatinal yüzeylerden 40 saniye süreyle polimerize edildi. Hastanın 1 yıllık takip süresi sonrasında restorasyonun başarılı bir şekilde ağızda durduğu, restorasyon ve diş arasında herhangi bir bağlantı problemi olmadığı, diş ve çevre dokuların sağlıklı olduğu görüldü (Resim 6).

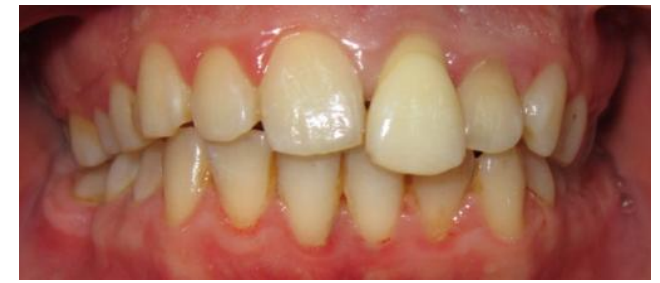

Resim 1- Hastanın ağız içi görüntüsü

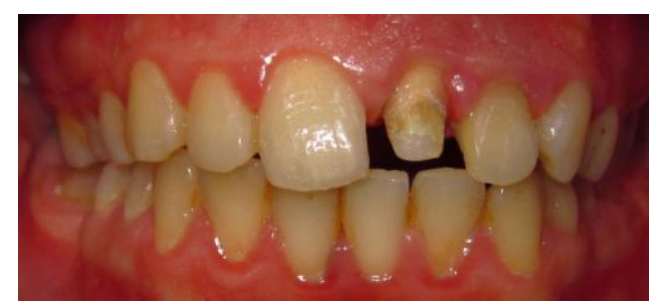

Resim 2- Prepare edilmiş diş

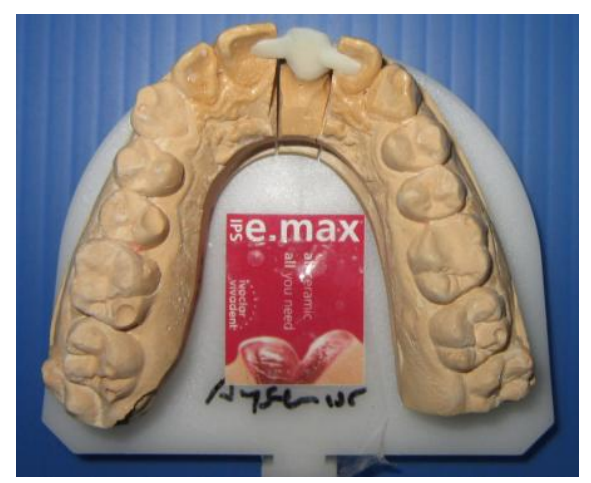

Resim 3- Modelde alt yapı görüntüsü

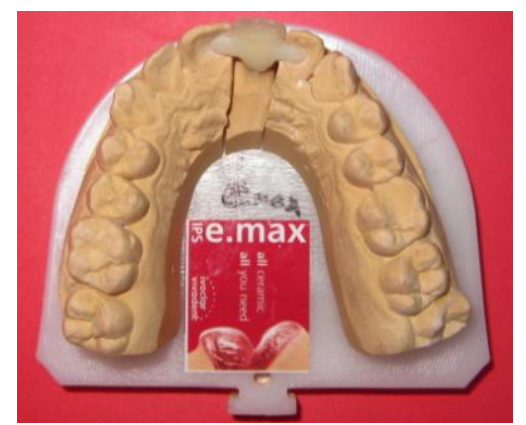

Resim 4- Modelde restorasyonun bitmiş hali

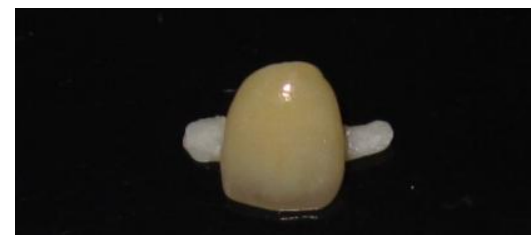

Resim 5- Hazırlanan restorasyonun görüntüsü

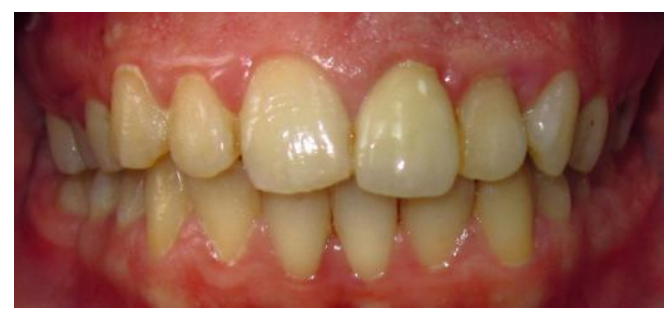

Resim 6- Protezin bitim aşaması

\section{TARTIŞMA}

$\mathrm{Bu}$ vaka raporunda santral kesici dişin estetik ve konservatif rehabilitasyonu anlatılmaktadır. Yapılacak olan protetik restorasyonlarda mevcut diş dokusunun korunması konservatif tedavi yaklaşımlarında önemli bir kriterdir. İyi bir tedavi planlaması ve doğru materyalin seçimi hazırlanacak restorasyonun başarısını etkilemektedir. ${ }^{5}$ Metal alt yapı içermeyen materyallerin kullanımı daha doğal ve estetik bir görünüm elde edilmesini sağlamaktadır. ${ }^{6}$

Biyouyumlulukları, esetik ve doğal görünümleri ve düşük plak birikimi özellikleri sayesinde tam seramik restorasyonlar klinikte başarılı bir şekilde kullanılmaktadır. Tam seramik restorasyonların uzun dönem klinik takipleri ile ilgili yapılmış pek çok çalışma bulunmaktadır ve bu çalışmaların sonuçları değerlendirildiğinde klinik açıdan başarılı oldukları bildirilmiştir. ${ }^{6,7}$ Özellikle cam seramik materyaller kullanılarak hazırlanan restorasyonların mekanik özellikleri sınırlı olduğundan adeziv simantasyon yapılması önerilmektedir ve bu sayede kırılma dayanıklılıklarının arttığı bildirilmiştir. $^{8,9}$

Simantasyon sırasında; porselen ile rezin siman arasındaki bağlantı ve diş ile rezin siman arasındaki bağlantı olmak üzere 2 bağlantı vardır. Adezyonun sağlanabilmesi için yapılacak olan simantasyon işlemi çok sayıda basamak içermektedir. Bağlantı dayanıklılığını arttırmak için restorasyonun iç yüzeyinin hidroflorik asit ile asitlenmesi ve silan uygulanması önerilmektedir. ${ }^{10,11}$

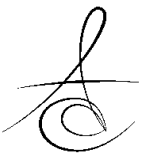


Seramik yüzeyi ve rezin siman arasında kimyasal bir reaksiyon olmadığında bağlantı sadece seramik yüzeyinden mekanik olarak sağlanacaktır. Silan uygulanması dental seramik ve rezin siman arasındaki kimyasal bağlantıyı sağlamak için kullanılmaktadır. ${ }^{12,13}$

Restorasyonun klinik olarak başarılı olmasında doğru endikasyon konulması, preparasyonun ideal bir şekilde yapılması, materyallerin üretici firma önerilerine uygun kullanılması ve simantasyon prosedürünün doğru bir şekilde uygulanması önemli kriterlerdir. ${ }^{14}$

İnley tutuculu kuron restorasyon klinik olarak uygulanımı kolay ve basit bir yöntemdir. İnvaziv tedavi seçeneklerini kabul etmeyen hastalar için alternatif bir tedavi yöntemi olarak önerilebilir.

\section{SONUÇ}

Hastanın üst anterior dişleri tam seramik restorasyon ile restore edilerek hem hasta hem de hekim için tatmin edici estetik sağlanmıştır. Tedavi sonrasında estetik ve fonksiyon açııından başarıı sonuçlar elde edilmiş ve hasta memnuniyeti sağlanmıştır.

\section{KAYNAKLAR}

1. Dilsiz A, Yağız H, Zihni M. Oklüzal travma: literatür derlemesi. Cumhuriyet Univ Dis Hek Fak Derg 2005;8:71-5.

2. Karakuş A, Tüfekçi ZI, Atalay Z. Agresif Periodontitis Vakasında Kombine Periodontal, Ortodontik ve İmplant Destekli Protetik Tedavi. Hacettepe Univ Dis Hek Fak Derg 2007;31:52-7.

3. Sasse M, Eschbach $S$, Kern M. Randomized clinical trial on single retainer all-ceramic resin-bonded fixed partial dentures: Influence of the bonding system after up to 55 months. J Dent 2012;40:783-6.

4. Kumbuloğlu O, Özdemir N, Aksoy G. A Different Pontic Design for Fiber-Reinforced Composite Bridgeworks: A Clinical Report. Eur J Dent 2007;1:50-3.

5. Karaalioglu O, Duymus ZY. Fiberle Güçlendirilmiş Kompozitlerin Sabit Bölümlü Protez Yapımında Kullanımları. Atatürk Üniv Diş Hek Fak Derg 2008;18:70-7.

6. Zimmer D, Gerds T, Strub JR. Survival rate of IPSEmpress 2 all-ceramic crowns and bridges: three year's results. Schweiz Monatsschr Zahnmed 2004;114:115-9.

7. Toksavul S, Toman M. A short term clinical evaluation of IPS Empress 2 crowns. Int J Prosthodont 2007;20:168-172.

8. McCormick JT, Rowland W, Shillingburg HT, Duncanson MG. Effect of luting media on the compressive strengths of two types of all-ceramic crowns. Quintessence Int 1993;24:405-8.

9. Burke FJ. The effect of variations in bonding procedure on fracture resistance of dentin-bonded all-ceramic crowns. Quintessence Int 1995;26:293300.

10. Ozcan M, Akkaya A. New approach to bonding allceramic adhesive fixed partial dentures: A clinical report. J Prosthet Dent 2002;88:252-4.

11. Iglesia-Puig MA, Arellano-Cabornero A. Inlay fixed partial denture as a conservative approach for restoring posterior missing teeth: a clinical report. J Prosthet Dent 2003;89:443-5.

12. Shen C, Oh WS, Williams JR. Effect of postsilanization drying on the bond strength of composite to ceramic. J Prosthet Dent 2004;91:453-8.

13. Hatakawa T, Horie K, Aida M, Kanaya H, Kobayashi $T$, Murata $Y$. The influence of surface conditions and silane agents on the bond of resin to dental porcelain. Dent Mater 1992;8:238-40.

14. Oilo G, Törnquist A, Durling $D$, Andersson M. Allceramic crowns and preparation characteristics: a mathematic approach. Int $\mathrm{J}$ Prosthodont 2003;16:301-6.

\author{
Yazışma Adresi \\ Dr. Ş. Begüm Türker \\ Marmara Üniversitesi \\ Diş Hekimliği Fakültesi \\ 34854 Başıbüyük, Maltepe \\ İstanbul, Türkiye \\ Tel: 02164121621 (1777) \\ Fax: 02164210291 \\ e-mail: begumturker @ hotmail.com
}

